\title{
Agenturen und Integrierte Kommunikation
}

\author{
Anforderungen an Agenturen aus Unternehmenssicht und deren Einfluss auf die \\ Umsetzung der Integrierten Kommunikation
}
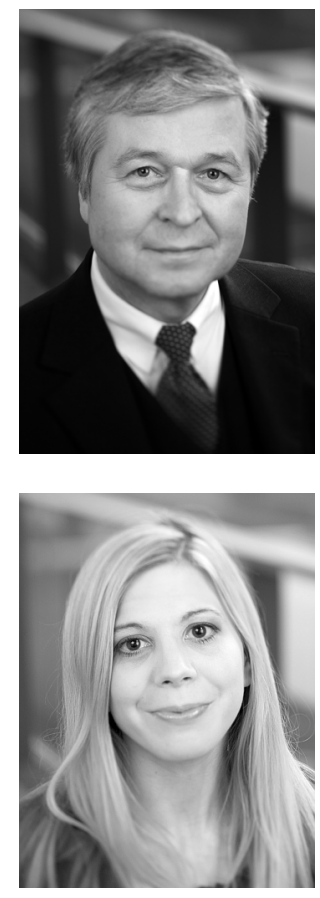

\section{Manfred Brubn und Verena Batt}

Kommunikationsagenturen, Integrierte Kommunikation, Kommunikationsfachabteilungen, Strukturgleichungsanalyse

Communications agencies, integrated communications, collaboration between companies and agencies, structural equation modeling

Bei der Umsetzung einer Integrierten Kommunikation fungieren Kommunikationsagenturen als externe Dienstleistungsanbieter. Oftmals sind jedoch den Agenturen die Anforderungen, die Unternehmen bei der Umsetzung der Integrierten Kommunikation an sie stellen, nicht bekannt - mit der Folge, dass Unzufriedenheit auf Seiten des Unternehmens entsteht. Bislang existiert noch keine Studie, die sich umfassend mit den Anforderungen an Agenturen im Rahmen der Integrierten Kommunikation beschäftigt. Das Ziel des Artikels besteht daher zum einen darin, diejenigen Anforderungen an Agenturen zu identifizieren, die Unternehmen im Rahmen der Umsetzung einer Integrierten Kommunikation an Agenturen stellen. Zum anderen besteht ein weiteres Ziel in der Ermittlung der Relevanz der Anforderungen für die Umsetzung der Integrierten Kommunikation.

Die Ergebnisse basieren auf der Durchführung qualitativer Experteninterviews und einer Strukturgleichungsanalyse. Letzterer liegt eine quantitative Befragung mit Kommunikations- und Marketingmanagern in schweizerischen Grossunternehmen zugrunde. Die Durchführung der Experteninterviews führen zur Identifikation von inhaltlichen und personellen Anforderungen an die Leistungsfähigkeit sowie inhaltlichen, organisatorischen und personellen Anforderungen an die Zusammenarbeit. Die aus der Strukturgleichungsanalyse resultierenden Ergebnisse unterstreichen die grosse Bedeutung der Erfüllung der inhaltlichen Anforderungen an die Zusammenarbeit und an die Leistungsfähigkeit für die Umsetzung der Integrierten Kommunikation. Darunter ist z. B. das Vorhandensein von Spezialistenwissen, das Angebot von Full-Service-Leistungen oder ein gleiches Verständnis von Integrierter Kommunikation zu subsumieren. Die Erfüllung organisatorischer Anforderungen (z. B. ein regelmässiger, institutionalisierter Informationsaustausch) ist den Ergebnissen zufolge für die Umsetzung der Integrierten Kommunikation von nachrangiger Bedeutung.

When implementing integrated communications, communications agencies often act as external service providers. These agencies are often not informed about the requirements that the companies contracting them expect, yielding unsatisfactory results for the companies. So far, no study, backed by a well-founded methodology, has yet investigated the requirements 
demanded of agencies for integrated communications. The goal of this article is therefore to identify which agency requirements are set by companies in the context of integrated communications implementation. Another goal is the investigation of the relevance of these requirements for the IMC implementation.

The results of this study are based on qualitative expert interviews and the use of structural equation modeling. The latter is based on a written survey of communications and marketing managers of large-scale Swiss enterprises. The expert interviews lead to the identification of content requirements set for performance capability, employee requirements set for performance capability, content requirements set for collaboration, organizational requirements set for collaboration and employee requirements set for collaboration. The findings of the structural equation modeling underscore the importance of satisfying the requirements that generate collaboration and fulfil achievement potential. Examples of such requirements are the presence of specialist knowledge, full-service performance and a shared understanding of integrated communications. The results show that the fulfillment of the organizational requirements (e.g., a regular, institutionalized exchange of information) is of subordinate importance for the successful implementation of integrated communications.

\section{Einleitung}

Das Konzept der Integrierten Kommunikation hat während der vergangenen zwei Jahrzehnten stetig an Bedeutung gewonnen (Schultz/Barnes 1999; Schultz 2003; Kitchen et al. 2004a; Kitchen et al. 2004b; Shimp 2007; Finne/Grönroos 2009). Veränderungen in den Kommunikations- und Medienmärkten sowie ein zunehmender Kostendruck in den Unternehmen haben in den letzten Jahren bei einer Vielzahl von Unternehmen zu der Einsicht geführt, dass nur durch eine konsequente Abstimmung der einzelnen Kommunikationsinstrumente eine kommunikationspolitische Profilierung zu erreichen ist, die das eigene Unternehmen bzw. die Marke(n) eigenständig von der Konkurrenz abgrenzt und in der Wahrnehmung der Zielgruppen verankert (Kessler 2004; Masker/Somosa 2004; Court et al. 2005; Elms 2007; Fasse 2007; Kitchen/Schultz 2009).

Bei der Umsetzung der Integrierten Kommunikation greifen Unternehmen häufig auf die Fähigkeiten von Kommunikationsagenturen zurück (Belch/Belch 2009; Moriarty et al. 2009). Dies sind oftmals klassische Werbeagenturen, PR-Agenturen oder Spezialagenturen mit einer bestimmten inhaltlichen Fokussierung. Diese Agenturen können die Unternehmen massgeblich bei der Koordination der Kommunikationsinstrumente und -massnahmen unterstützen (Gronstedt 1996). Allerdings sind oftmals Schwierigkeiten mit der Umsetzung von integrierten Kommunikationskonzepten durch Agenturen verbunden (Caywood et al. 1991; Davidson/Ewing 1997; Schultz/Kitchen 1997; Kitchen/Schultz 1999; Low 2000; Kitchen et al. 2008). Dies äussert sich z. B. darin, dass die beauftragten Agenturen nicht die erforderliche Fachkompetenz in Bezug auf die zu integrierenden Kommunikationsinstrumente aufweisen (Schultz/Kitchen 1997). Eine weitere Schwierigkeit entsteht dadurch, dass von einem Unternehmen unterschiedliche Agenturen mit der Integrierten Kommunikation beauftragt werden. Diese Agenturen konkurrieren häufig miteinander, indem sie z. B. einen möglichst grossen Etatanteil für das „eigene“ Kommunikationsinstrument zu erkämpfen anstreben, anstatt an der gemeinsamen Aufgabe der Umsetzung einer Integrierten Kommunikation zu arbeiten. Verfügen die Agenturen zudem über eine unterschiedliche Organisationsstruktur bzw. Unternehmenskultur, wirkt sich dies ebenfalls erschwerend auf die Umsetzung der Integrierten 
Kommunikation aus (Kitchen et al. 2008). Die bestehenden Schwierigkeiten sind darüber hinaus darauf zurückzuführen, dass Unkenntnis bzgl. der Rollen- und Aufgabenverteilung bei Agenturen besteht oder Unstimmigkeiten bzgl. der Auswahl der zu integrierenden Kommunikationsinstrumente vorliegen (Schultz et al. 1994; Beard 1996; Cathey 1996; Gould et al. 1999). Die Folge ist eine mangelhafte Umsetzung der Integrierten Kommunikation durch den Einsatz der Agentur, verbunden mit der Entstehung von Unzufriedenheit auf Seite des Unternehmens. Um dies zu vermeiden, ist die Kenntnis der Anforderungen, die Unternehmen als Auftraggeber im Rahmen der Planung und Umsetzung der Integrierten Kommunikation an Agenturen stellen, von Relevanz. Diese Kenntnis stellt die Basis für eine effektive Umsetzung der Integrierten Kommunikation mithilfe von Agenturen dar (vgl. ähnlich Eagle/Kitchen 2000), und es besteht auf beiden Seiten ein Bewusstsein darüber, welche Aufgaben wer zu übernehmen hat und für welche jeweils die „andere Seite“ zuständig ist.

Bislang existiert aber noch keine Studie, die sich ausführlich mit den Anforderungen an Agenturen im Rahmen der Umsetzung der Integrierten Kommunikation beschäftigt. Es liegen zwar Studien zur Zusammenarbeit zwischen Unternehmen und Agenturen im Kontext der Integrierten Kommunikation vor, diese beschäftigen sich aber zumeist nur am Rande mit den Anforderungen an Agenturen (z. B. Schultz/Kitchen 1997). Das Ziel des vorliegenden Artikels ist daher die Schliessung dieser Forschungslücke, indem es gilt, die Anforderungen, die Unternehmen im Rahmen der Umsetzung der Integrierten Kommunikation an Agenturen stellen, zu identifizieren. Ferner ist zu prüfen, welche Relevanz der Erfüllung dieser Anforderungen zur Umsetzung der Integrierten Kommunikation zukommt. Hierzu werden qualitative Experteninterviews sowie eine schriftliche Befragung mit Kommunikations- und Marketingleitern der 500 umsatzstärksten Schweizer Unternehmen durchgeführt.

Der Artikel gliedert sich in sechs Teile: In Kapitel 2 werden die Grundlagen der Integrierten Kommunikation diskutiert. Anschliessend wird in Kapitel 3 der Stand der Literatur zu Anforderungen an Agenturen im Rahmen der Umsetzung der Integrierten Kommunikation aufgezeigt. Dies mündet in die Ableitung der zentralen Forschungsfragen. Kapitel 4 widmet sich der Identifikation der Anforderungen an Agenturen. Hierzu werden die Ergebnisse der qualitativen Experteninterviews präsentiert. In Kapitel 5 erfolgt die Entwicklung und Prüfung des Untersuchungsmodells, um die Relevanz der Erfüllung der identifizierten Anforderungen zu ermitteln. Kapitel 6 bildet das Fazit mit der Ableitung von Praxisimplikationen und einer kritischen Würdigung der Untersuchungsergebnisse.

\section{Grundlagen zur Integrierten Kommunikation}

Bislang besteht weder in der Wissenschaft noch in der Praxis Einigkeit hinsichtlich einer eindeutigen Definition des Konzepts der Integrierten Kommunikation (Kerr et al. 2008; Kliatchko 2008; Kitchen/Schultz 2009). Dieser fehlende definitorische Konsens führt wiederkehrend zu Diskussionen über die Bedeutung des Konzepts (z. B. Kitchen/Schultz 1999; Cornelissen/Lock 2000; Low 2000; Duncan/Mulhern 2004; Kliatchko 2005), da letztlich sowohl die Entwicklung einer Theorie als auch die Messung des Erfolgs einer Integrierten Kommunikation eine eindeutige Definition voraussetzen. Um im Rahmen der vorliegenden Umfrage ein einheitliches Begriffsverständnis zugrunde zu legen, wurde sich für die Studie auf folgende Definition der Integrierten Kommunikation geeinigt:

„Integrierte Kommunikation ist ein strategischer und operativer Prozess der Analyse, Planung, Organisation, Durchführung und Kontrolle, der darauf ausgerichtet ist, aus den 
differenzierten Quellen der internen und externen Kommunikation von Unternehmen eine Einheit herzustellen, um ein für die Zielgruppen der Kommunikation konsistentes Erscheinungsbild des Unternehmens bzw. eines Bezugsobjektes der Kommunikation zu vermitteln." (Brubn 2009, 22)

Im Rahmen des Konzepts der Integrierten Kommunikation wird zwischen drei verschiedenen Formen der Integration unterschieden: die inhaltliche, formale und zeitliche Integration (Brubn 2009, 80ff.).

Ein zentraler Schwerpunkt der Integrierten Kommunikation stellt die inhaltliche Integration, d. h. die thematische Abstimmung aller Kommunikationsinstrumente und -mittel, dar. Dies erfolgt durch verschiedene Verbindungslinien, beispielsweise die Verwendung eines einheitlichen Slogans, Kernbotschaften und -argumente, Schlüsselbilder sowie auch akustische Signale u. a. m. Das Ziel der inhaltlichen Integration stellt die Vermittlung eines einheitlichen Erscheinungsbildes dar, aus der inhaltliche Aussagen über den Absender entnommen werden können.

Unter formaler Integration wird die Vereinheitlichung unterschiedlicher Kommunikationsmittel verstanden. Die Verbindung der Instrumente und Mittel erfolgt oftmals unter $\mathrm{Zu}$ hilfenahme von bestimmten Gestaltungsprinzipien, z. B. die Verwendung einheitlicher Unternehmens- bzw. Markenzeichen, Logos sowie gleicher Schrifttypen, -farben und -grössen usw. Durch eine konsequente Einhaltung solcher Gestaltungsprinzipien auf horizontaler (instrumenteübergreifend) sowie vertikaler (zielgruppenübergreifend) Ebene stellen Unternehmen ein einheitliches Erscheinungsbild des kommunikativen Bezugsobjektes sicher. Dieses zielt auf die leichte Wiedererkennbarkeit, das Realisieren von Lerneffekten bei den Zielgruppen sowie auf die Verankerung der Marke im Gedächtnis der Rezipienten ab.

Im Rahmen der zeitlichen Integration sind sämtliche Kommunikationsaktivitäten, -instrumente und -mittel innerhalb und zwischen verschiedenen Planungsperioden abzustimmen. Ein Teilaspekt betrifft die zeitliche Abstimmung zwischen verschiedenen Kommunikationsinstrumenten, um die gegenseitige Unterstützung im zeitlichen Einsatz sicherzustellen. Des Weiteren ist die zeitliche Kontinuität innerhalb eines Kommunikationsinstrumentes zu gewährleisten. Demnach sind Wechsel der Kommunikationskonzepte zu vermeiden, um bei den Rezipienten Wiederholungs- und Lerneffekte der Kommunikationsinhalte zu generieren.

\section{Literaturreview zu Anforderungen an Agenturen bei der Umsetzung der Integrierten Kommunikation}

Zur Zusammenarbeit von Unternehmen und Agenturen im Rahmen von Aktivitäten der Integrierten Kommunikation liegen zwar einige Beiträge vor (z. B. Duncan/Everett 1993; Beard 1996; Gronstedt 1996; Schultz/Kitchen 1997; Gould et al. 1999; Eagle/Kitchen 2000; Kim et al. 2004; Swain 2004; Kitchen et al. 2007; Kitchen et al. 2008; Kerr/Drennan 2010). Nach Kenntnis der Autoren finden sich hingegen bis auf den Beitrag von Gould et al. (1999) keine Studien, die explizit die Anforderungen an Agenturen bei der Umsetzung der Integrierten Kommunikation untersuchen. In einigen wenigen Beiträgen werden aber Barrieren einer Integrierten Kommunikation aufgezeigt, die auf mögliche Anforderungen an Agenturen schliessen lassen (z. B. bei Duncan/Everett 1993; Eagle/Kitchen 2000). Auf diese wird, neben der Studie von Gould et al., im Folgenden eingegangen. 
Gould et al. (1999) leiten aus den in der Literatur berichteten Ergebnissen früherer Studien Anforderungen an Agenturen und Unternehmen ab, deren Erfüllung die Voraussetzung für die effektive Umsetzung einer Integrierten Kommunikation darstellen soll. Die Autoren beziehen sich sowohl auf Unternehmen als auch auf Agenturen und identifizieren folgende Anforderungen an die beiden Akteure: ein beidseitiges Commitment gegenüber dem Konzept der Integrierten Kommunikation, ein regelmässiger Kontakt zwischen Agentur und Unternehmen, eine offene Kommunikation, ein proaktiver Wissensaustausch, das Vorhandensein von Kommunikationsschnittstellen (z. B. durch Informationstechnologien), eine Beziehung zwischen den Akteuren, die auf Vertrauen und Commitment basiert und eine geringe Anzahl an Agenturen, die mit dem Unternehmen zusammenarbeiten. Unternehmen und Agenturen sollten nach Ansicht der Autoren ausserdem zueinander passen. Die Forscher gehen in ihrem Beitrag ausführlich auf die einzelnen Anforderungen ein und leiten Handlungsempfehlungen ab. Eine Untermauerung der aus der Literatur identifizierten Anforderungen durch Interviews mit Unternehmensvertretern sowie die empirische Überprüfung, inwieweit die Anforderungen einen Einfluss auf die Umsetzung der Integrierten Kommunikation haben, bleiben jedoch aus.

Duncan und Everett (1993) befragten US-Kommunikations- und Marketingmanager zur Wahrnehmung und Organisation der Integrierten Kommunikation im Unternehmen. Unter anderem wurde dabei die Frage nach den Barrieren einer Integrierten Kommunikation gestellt. Die Ergebnisse zeigen, dass zum einen interne Barrieren existieren, die z. B. aus „Grabenkämpfen “ zwischen Kommunikationsabteilungen bestehen. Zum anderen liegen jedoch auch externe Barrieren vor, die hauptsächlich aus „agency egos“ resultieren. Die Autoren gehen nicht genauer auf die Bedeutung dieses Sachverhalts ein; es ist aber zu vermuten, dass unter „Agenturegoismen“ Aspekte, wie z. B. eine aus Know-how- oder Kostengründen fehlende Neutralität bei der Auswahl der Kommunikationsinstrumente für Unternehmen zu verstehen sind.

Eagle und Kitchen (2000) führen zu Fragestellungen der Integrierten Kommunikation Interviews mit Agentur- und Unternehmensvertretern aus Neuseeland durch. Die Befragung zeigt, dass sich ein zu grosser Egoismus auf beiden Seiten, der zu Lasten der Professionalität geht, negativ auf die Umsetzung der Integrierten Kommunikation auswirkt. Als bedeutsam werden hingegen die Teamarbeit zwischen den Akteuren sowie die kontinuierliche Weiterbildung der Akteure zu Fragen der Integrierten Kommunikation angesehen.

Wie aus dem Literaturüberblick hervorgeht, liegen bisher kaum Erkenntnisse über Anforderungen an Agenturen im Rahmen der Integrierten Kommunikation vor. Duncan/ Everett (1993) sowie Eagle/Kitchen (2000) gehen lediglich auf die Barrieren einer Integrierten Kommunikation ein, konkrete Anforderungen an Agenturen werden vernachlässigt und lassen sich bestenfalls aus den genannten Barrieren folgern. Gould et al. (1999) führen immerhin einige Anforderungen auf, die eine Umsetzung der Integrierten Kommunikation fördern; diese erfahren jedoch keine empirische Überprüfung. Zudem ist es fraglich, ob die identifizierten Anforderungen umfassend sind, da sie lediglich eine Ableitung aus Erkenntnissen von Studien darstellen, die nicht zum Ziel hatten, die zentralen Anforderungen an Agenturen im Rahmen der Integrierten Kommunikation zu ermitteln.

Diese Forschungslücken geben an dieser Stelle Anlass, folgende Forschungsfragen abzuleiten, die nachfolgend mittels der Erkenntnisse zweier Studien beantwortet werden sollen: 
(1) „Welche sind die Anforderungen, die Unternehmen im Rabmen der Umsetzung einer Integrierten Kommunikation an Agenturen stellen?"

$\rightarrow$ Identifikation von Anforderungen durch Studie 1 (Kapitel 4)

(2) „Welche Relevanz kommt der Erfüllung dieser Anforderungen für die Umsetzung einer Integrierten Kommunikation zu?" “

$\rightarrow$ Ermittlung der Relevanz der Erfüllung der Anforderungen durch Studie 2 (Kapitel 5)

\section{Identifikation von Anforderungen an Agenturen bei der Umsetzung der Integrierten Kommunikation (Studie 1)}

\subsection{Methodische Vorgehensweise}

Für die Beantwortung der ersten Forschungsfrage werden 20 qualitative Interviews mit Experten aus Unternehmen, die mit Agenturen bei der Planung und Umsetzung der Integrierten Kommunikation zusammenarbeiten, durchgeführt (Studie 1). Die Interviews dauern jeweils etwa eine Stunde und werden mittels eines Interviewleitfadens gestützt. Dabei kommen ausschliesslich offene Fragen zum Einsatz. Das Ziel der Experteninterviews ist es, jene Anforderungen an Agenturen zu identifizieren, die aus Unternehmenssicht einen Beitrag zur Umsetzung der Integrierten Kommunikation leisten.

$\mathrm{Zu}$ Beginn der Interviews werden zunächst Fragen bezüglich des allgemeinen Verständnisses einer Integrierten Kommunikation gestellt. Diese dienen als „Eisbrecherfragen“ und haben das Ziel zu prüfen, inwieweit das in Kapitel 2 zugrunde gelegte Verständnis der Integrierten Kommunikation in der Unternehmenspraxis Gültigkeit hat. Die Befragten geben ausnahmslos an, dass sie mit der Integration der Kommunikation eine Abstimmung der Kommunikation in inhaltlicher und formaler Hinsicht verbinden. Die zeitliche Integration wird nicht von allen genannt, bei einem Nachfragen von Seiten des Interviewers bestätigen jedoch sämtliche Befragten die Zugehörigkeit der zeitlichen Integration zur Integrierten Kommunikation.

Im Anschluss an die Schaffung eines gemeinsamen Grundverständnisses der Integrierten Kommunikation werden im Hauptteil der Interviews Fragen zur eigentlichen Zielsetzung der Studie gestellt. In diesem Zusammenhang werden die Befragten gebeten, sämtliche Anforderungen an Agenturen zu nennen, die die Umsetzung der Integrierten Kommunikation durch den Einsatz von Agenturen beeinflussen. In Tabelle 1 sind die angeführten Anforderungen an Agenturen dargestellt. 
Anforderungen der Unternehmen an Agenturen

- Angebot eines breiten Leistungsspektrums (Full-Service-Leistungen)

- Vorhandensein von Spezialistenwissen (in Bezug auf einzelne Kommunikationsinstrumente wie z. B. Social Media)

- Vorhandensein von Generalistenwissen (in Bezug auf sämtliche Kommunikationsinstrumente)

- Schnelligkeit der Umsetzung der Integrierten Kommunikation durch die Agentur

- Fähigkeit hinsichtlich der strategischen Planung der Integrierten Kommunikation (strategische Fähigkeiten)

- Fähigkeit hinsichtlich der praktischen Vernetzung von Kommunikationsinstrumenten (operative Fähigkeiten)

- Generierung von Synergien zwischen einzelnen Kommunikationsinstrumenten/Verbindung der eingesetzten Kommunikationsinstrumente

- Bereitschaft der Agenturmitarbeitenden, das Unternehmen in Bezug auf Fragestellungen der Integrierten Kommunikation zu unterstützen

- Interesse der Agenturmitarbeitenden in Bezug auf die Aneignung von Wissen und Know-how zu Fragestellungen der Integrierten Kommunikation

- Fit zwischen Unternehmen und Agentur im Hinblick auf das Verständnis von Integrierter Kommunikation

- Kenntnis der Agentur über die Ziele des Unternehmens

- Bewusstsein der Agentur im Hinblick auf die Bedeutung der Integrierten Kommunikation für das Unternehmen

- Leistungsnachweis der Agentur im Rahmen der Umsetzung der Integrierten Kommunikation

- Einbindung der internen Kommunikation des Unternehmens im Rahmen der Umsetzung der Integrierten Kommunikation

- Regelmässige Zusammenarbeit und Abstimmung zwischen Unternehmen und Agentur

- Regelmässiger Informationsaustausch zwischen Unternehmen und Agentur

- Vorliegen schriftlich festgehaltener Abstimmungs- und Entscheidungsregeln, z. B. in der Form von Milestones

- Örtliche Nähe zwischen Unternehmen und Agentur

- Gleiches Verständnis im Hinblick auf die Rolle der Agentur bei der Umsetzung der Integrierten Kommunikation

- Bereitschaft der Agentur, sich mit dem Unternehmen abzustimmen und zusammenzuarbeiten

- Offenheit und Vertrauenswürdigkeit im Umgang mit dem Unternehmen

Personelle Anforderungen an die Leistungsfähigkeit

Inhaltliche Anforderungen an die

Leistungsfähigkeit

Inhaltliche Anforderungen an die Zusammenarbeit

Organisatorische Anforderungen an die

Zusammenarbeit

Personelle Anforderungen an die Zusammenarbeit

- Kontinuierlicher Ansprechpartner auf Agenturseite

- Flexibilität der Agentur, z. B. bei Änderungswünschen des Unternehmens

- Zuverlässigkeit der Agentur (z. B. im Hinblick auf Termineinhaltung) 
Wie aus der Tabelle hervorgeht, liegt eine Vielzahl von Anforderungen, die Unternehmen an Agenturen bei der Umsetzung der Integrierten Kommunikation stellen, vor. Die einzelnen Anforderungen lassen sich nach ihrer inhaltlichen Ähnlichkeit bündeln, so dass insgesamt fünf verschiedene Formen von Anforderungen an Agenturen identifiziert werden können. Im Rahmen der Interviews wird zum einen die Bedeutung der Anforderungen an die Leistungsfähigkeit der Agenturen ersichtlich. Hierbei erfolgt eine Unterscheidung nach inhaltlichen und personellen Anforderungen an die Leistungsfähigkeit. Erstere nehmen Bezug auf die Fähigkeiten der Agentur, das Unternehmen im Rahmen der Planung und Umsetzung der Integrierten Kommunikation zu unterstützen, und beinhalten z. B. das Vorhandensein von Generalisten- und Spezialistenwissen in Bezug auf die vom Unternehmen eingesetzten Kommunikationsinstrumente. Letztere beziehen sich auf Erwartungen in personeller Hinsicht, die für die Leistungserbringung im Hinblick auf die Integrierte Kommunikation von Agenturseite zu erfüllen sind. Darunter ist z. B. die Bereitschaft der Mitarbeitenden, sich Wissen über die Integrierte Kommunikation anzueignen, zu subsumieren. Zum anderen werden in den Interviews Anforderungen an die Zusammenarbeit deutlich. Diese lassen sich nach inhaltlichen, organisatorischen und personellen Anforderungen an die Zusammenarbeit unterscheiden. Im Gegensatz zu den Konstrukten der Leistungsfähigkeit, die auf leistungsbezogene Aspekte Bezug nehmen, rekurrieren die Konstrukte zur Zusammenarbeit mit Agenturen auf die Interaktion zwischen Unternehmen und Agentur. Die Zusammenarbeit in inhaltlicher Hinsicht manifestiert sich z. B. im gleichen Verständnis von Integrierter Kommunikation, in organisatorischer Hinsicht besteht z. B. die Erwartung nach einer regelmässigen Abstimmung zwischen Unternehmen und Agentur. Die personellen Anforderungen an die Zusammenarbeit drücken sich z. B. in der Erwartung nach einem kontinuierlichen Ansprechpartner auf Seiten der Agentur oder nach einem offenen, vertrauenswürdigen Umgang aus.

Die in den Interviews angeführten Anforderungen stimmen teilweise mit den in der Literatur aufgezeigten Barrieren einer Integrierten Kommunikation bei der Zusammenarbeit mit Agenturen überein. So wird in der Literatur insbesondere auf die Anforderungen an die Zusammenarbeit verwiesen (z. B. eine offene Kommunikation, ein regelmässiger Kontakt zwischen Unternehmen und Agentur; vgl. Kapitel 3). Ein zentraler Unterschied besteht hingegen in Bezug auf die Anforderungen an die Leistungsfähigkeit. Während darauf in der Literatur kaum eingegangen wird, stellen die Befragten zahlreiche Anforderungen an die Leistungsfähigkeit der Agentur, insbesondere in inhaltlicher Hinsicht.

Zusammenfassend führt die Durchführung der qualitativen Interviews zur Identifikation folgender Anforderungen:

- Inhaltliche Anforderungen an die Leistungsfähigkeit,

- Personelle Anforderungen an die Leistungsfähigkeit,

- Inhaltliche Anforderungen an die Zusammenarbeit,

- Organisatorische Anforderungen an die Zusammenarbeit und

- Personelle Anforderungen an die Zusammenarbeit.

\section{5. Überprüfung der Relevanz der Anforderungen für die Umsetzung der Integrierten Kommunikation (Studie 2)}

\subsection{Entwicklung eines Strukturgleichungsmodells und Hypothesengenerierung}

Die Identifikation der Anforderungen an Agenturen bei der Umsetzung der Integrierten Kommunikation dient als Basis für die Beantwortung der zweiten Forschungsfrage. Hier gilt 
es zu prüfen, welche Relevanz der Erfüllung der identifizierten Anforderungen für die Umsetzung der Integrierten Kommunikation in Unternehmen jeweils zukommt. Für die Ermittlung der jeweiligen Relevanz ist ein Strukturgleichungsmodell zu entwickeln, das auf Basis einer quantitativen Umfrage einer empirischen Überprüfung unterzogen wird (Studie 2).

Das hier zu entwickelnde Strukturgleichungsmodell setzt die Erfüllung der identifizierten Anforderungen (Erfüllung der inhaltlichen und personellen Anforderungen an die Leistungsfähigkeit, Erfüllung der inhaltlichen, organisatorischen und personellen Anforderungen an die Zusammenarbeit) in Beziehung zur Umsetzung der Integrierten Kommunikation durch den Einsatz der Agentur. Die Erfüllung der Anforderungen sowie die Umsetzung der Integrierten Kommunikation stellen dabei hypothetische Konstrukte dar, die sich einer direkten Messbarkeit entziehen (Bagozzi/Fornell 1982; Bagozzi/Philipps 1982). Es besteht daher die Notwendigkeit einer Konstruktoperationalisierung, indem es für jedes Konstrukt beobachtbare Indikatoren zu finden gilt (Churchill 1979; Homburg/Giering 1996). Für die Messbarmachung der Anforderungskonstrukte dienen die Erkenntnisse aus den zuvor dargestellten qualitativen Interviews. Es werden jeweils die den Konstrukten zugeordneten Anforderungen als Messindikatoren berücksichtigt. Die Operationalisierung des Konstrukts Umsetzung der Integrierten Kommunikation erfolgt unter Bezugnahme auf das dieser Studie zugrunde liegende Verständnis von Integrierter Kommunikation und berücksichtigt mit insgesamt sieben Indikatoren die inhaltliche, formale und zeitliche Integration durch den Einsatz der Agentur (vgl. Kapitel 2). Tabelle 4 im Anhang zeigt einen Überblick über die Konstrukte und die dazugehörigen Messindikatoren.

Es ist anzumerken, dass sämtliche Modellkonstrukte reflektiv operationalisiert werden. Der Grund hierfür ist die Überlegung, dass die einzelnen Indikatoren die Konstrukte widerspiegeln. So wird z. B. postuliert, dass mit der wahrgenommenen Erfüllung der Anforderungen durch die Agentur eine positive Bewertung der einzelnen Indikatoren verbunden wird. Zudem liegt die Annahme einer Abhängigkeit bzw. Korreliertheit der einzelnen Indikatoren eines Konstrukts zugrunde (Jarvis et al. 2003).

Sämtliche Modellkonstrukte werden zudem eindimensional operationalisiert. Dies gilt auch für das Konstrukt Umsetzung der Integrierten Kommunikation durch den Einsatz der Agentur, da die Annahme besteht, dass die Formen der Integrierten Kommunikation (siehe Kapitel 2) - inhaltliche, formale und zeitliche Integration - nicht ausreichend diskriminieren.

Anschliessend werden die Konstrukte in weiteren Experteninterviews auf Vollständigkeit, Redundanz und Verständlichkeit überprüft. Dabei gilt es für die Befragten, alle generierten Items den jeweiligen Konstrukten zuzuteilen. Es zeigt sich, dass die Zuordnung der Indikatoren bei jedem der fünf Konstrukte exakt der ursprünglichen Zuordnung entspricht. Aufgrund der ihnen zugeschriebenen Relevanz werden zudem sämtliche Indikatoren beibehalten.

In Abbildung 1 ist das entwickelte Strukturgleichungsmodell grafisch dargestellt. 


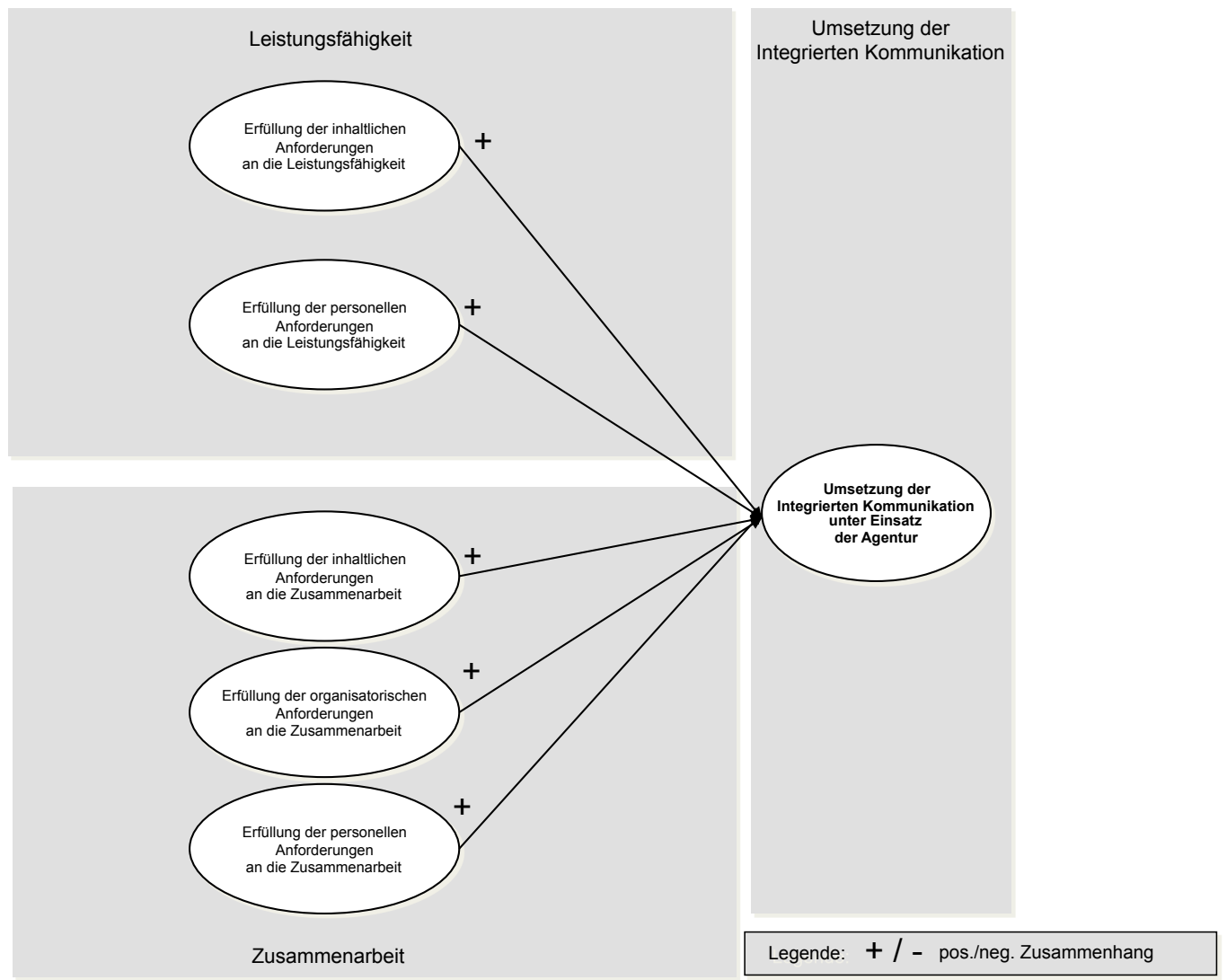

Abbildung 1: Konzeptualisiertes Strukturgleichungsmodell

Konkret ergeben sich daraus für die Untersuchung folgende Einzelhypothesen (vgl. Tabelle 2):

\section{Hypothesen}

Je mehr die Agentur die inhaltlichen Anforderungen an die Leistungsfähigkeit erfüllt, $H_{1}$ : $\quad$ desto stärker erfolgt die Umsetzung der Integrierten Kommunikation durch den Einsatz der Agentur.

Je mehr die Agentur die personellen Anforderungen an die Leistungsfähigkeit erfüllt,

$\mathrm{H}_{2}$ : $\quad$ desto stärker erfolgt die Umsetzung der Integrierten Kommunikation durch den Einsatz der Agentur.

Je mehr die Agentur die inhaltlichen Anforderungen an die Zusammenarbeit erfüllt,

H3: $\quad$ desto stärker erfolgt die Umsetzung der Integrierten Kommunikation durch den Einsatz der Agentur. 
Hypothesen

Je mehr die Agentur die organisatorischen Anforderungen an die Zusammenarbeit er-

$H_{4}$ : $\quad$ füllt, desto stärker erfolgt die Umsetzung der Integrierten Kommunikation durch den Einsatz der Agentur.

Je mehr die Agentur die personellen Anforderungen an die Zusammenarbeit erfüllt, H5: $\quad$ desto stärker erfolgt die Umsetzung der Integrierten Kommunikation durch den Einsatz der Agentur.

Tabelle 2: Darstellung der Hypothesen

\subsection{Fragebogenkonzeption und Datenerhebung}

Für die Datenerhebung werden als Grundgesamtheit Kommunikations- bzw. MarketingleiterInnen der jeweils 500 umsatzstärksten Unternehmen in der Schweiz definiert, auf deren Basis die Durchführung einer Vollerhebung erfolgte. Als Datenerhebungsverfahren findet eine schriftliche Befragung mittels postalisch versandten Fragebögen statt.

Die Fragebögen gliedern sich in drei Teile: Im ersten Teil werden allgemeine Daten zur Unternehmenskommunikation abgefragt. Der zweite Teil widmet sich der Zusammenarbeit der Unternehmen mit Agenturen, wobei hier neben allgemeinen Eckdaten zur Kooperation ebenso die Bewertung der konstruktbildenden Indikatoren entlang einer siebenstufigen Likert-Skala (von 1 = stimme voll und ganz zu bis 7 = stimme überhaupt nicht zu) erbeten wird. Im dritten Teil werden allgemeine Angaben zum Unternehmen abgefragt.

Die Umfrage erreicht mit dem Erhalt von 135 vollständig ausgefüllten Fragebögen eine Rücklaufquote von knapp 30 Prozent. Die adressierten Unternehmen stammen dabei zu 48,5 Prozent aus der Dienstleistungsbranche, gefolgt von Industriegüterherstellern (15,9 Prozent), Verbrauchsgüterherstellern (15,2 Prozent) und Gebrauchsgüterherstellern (14,4 Prozent). Öffentliche Unternehmen beteiligten sich zu 6,1 Prozent an der Befragung.

\subsection{Datenanalyse}

Für die Auswertung der unternehmensseitigen Anforderungen an Agenturen und deren Einfluss auf die Umsetzung der Integrierten Kommunikation wird eine Strukturgleichungsanalyse mithilfe des Analyseprogramms PLS Graph 3.0 durchgeführt. Zur Beurteilung von varianzbasierten PLS-Ergebnissen liegt noch kein geeignetes globales Gütemass vor. Zur Abschätzung der Modellgüte, und damit zur Sicherstellung der Zuverlässigkeit des Gesamtmodells, erfolgt daher in dieser Studie eine Orientierung an den von Chin et al. vorgeschlagenen Prüfkriterien (Chin 1998b; Chin/Newsted 1999; für eine Übersicht über die Prüfkriterien für PLS-Modelle vgl. Herrmann et al. 2006).

Wie bereits aufgezeigt, sind sämtliche Messmodelle reflektiver Art. Zur Validierung der Messmodelle kommt zunächst die exploratorische Faktorenanalyse (EFA) zum Einsatz. Diese untersucht eine grössere Anzahl an Indikatoren auf die ihnen zugrunde liegende Faktorenstruktur mit dem Ziel, die Indikatoren zu möglichst wenigen Faktoren zu verdichten. Hierbei wird gefordert, dass ein Faktor mindestens 50 Prozent der Varianz der ihm zugehörigen Indikatoren erklärt (Homburg/Giering 1996). Ein weiteres Gütemass ist das Cronbach'sche Alpha. Hier wird ein Schwellenwert von 0,7 gefordert (Nunnally 1978). Für die Konstruktreliabilität (KR) und die durchschnittlich erfasste Varianz (DEV) wird ein Schwellenwert von 0,6 (KR) (Bagozzi/Yi 1988) bzw. 0,5 (DEV) (Fornell/Larcker 1981) festgelegt. 
Für die Faktorladungen wurde ein Mindestwert von 0,5 gefordert. Die dazugehörigen TWerte verlangen für das Vorliegen von Signifikanz einen Mindestwert von 1,64 (Chin 1998 b; Chin/Newsted 1999).

Im Rahmen der Beurteilung des Strukturmodells interessiert die Erklärung der Zielvariablen, hier der Umsetzung der Integrierten Kommunikation durch den Einsatz der Agentur, durch die exogenen Variablen. Dies erfolgt über das Bestimmtheitsmass $\mathrm{R}^{2}$. Fällt dieser Wert ausreichend hoch (z. B. $\geq 0,3$, Chin 1998 b) aus, ist dies ein Zeichen für einen guten Einbezug der relevanten Anforderungen der Umsetzung der Integrierten Kommunikation durch den Einsatz der Agentur. Bei den Pfadkoeffizienten sind Werte unter 0,2 zu vernachlässigen (Chin 1998 a). Die Signifikanz der Pfadkoeffizienten wird über T-Statistiken berechnet.

\subsection{Empirische Ergebnisse}

Nachfolgend wird zunächst auf die Überprüfung der Messmodelle eingegangen (vgl. für eine detaillierte Darstellung der Ergebnisse Tabelle 4 im Anhang). Anschliessend werden die Ergebnisse des Strukturmodells vorgestellt, die Aufschluss über den Einfluss der Erfüllung der Anforderungen auf die Umsetzung der Integrierten Kommunikation durch den Einbezug der Agenturen geben. Abbildung 2 zeigt die Untersuchungsergebnisse im Überblick.

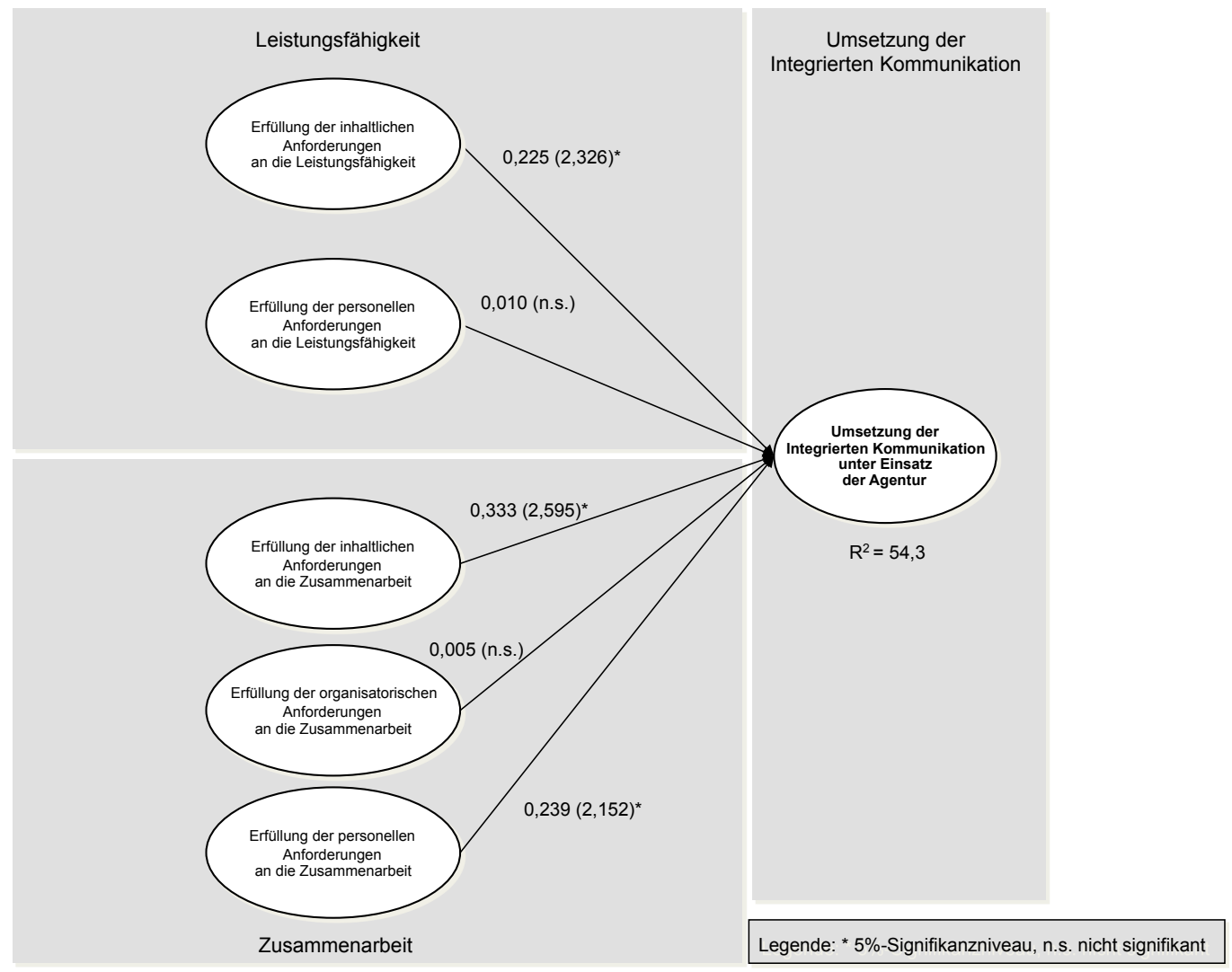

Abbildung 2: Ergebnisse des Strukturgleichungsmodells 
Die Überprüfung des Konstrukts Erfüllung der inhaltlichen Anforderungen an die Leistungsfähigkeit weist zunächst auf eine mangelnde Güte des Messmodells hin. So ergibt sich aus der exploratorischen Faktorenanalyse ein Varianzerklärungsbeitrag, der deutlich unter den geforderten 50 Prozent liegt. Da für die Faktorladung des Items Vorhandensein von Generalistenwissen eine deutliche Unterschreitung des Mindestwerts von 0,5 zu verzeichnen ist, wird sich für die Eliminierung dieses Items entschieden. Durch die Eliminierung können für das Messmodell vertretbare Werte erzielt werden. Die leichte Unterschreitung des Varianzerklärungsbeitrags sowie des Werts der durchschnittlich erfassten Varianz wird als tolerierbar angesehen. Eine mögliche Begründung für die niedrige Faktorladung im Hinblick auf das Vorhandensein von Generalistenwissen liefert die Messskala. Es ist möglich, dass die Befragten die Items Vorhandensein von Generalistenwissen und Spezialistenwissen als sich gegenseitig ausschliessend betrachtet haben und sich daher für das Item entschieden, das stärker in Verbindung mit der Erfüllung der inhaltlichen Anforderungen an die Leistungsfähigkeit gebracht wurde (in diesem Falle das Spezialistenwissen).

Im Rahmen der Untersuchung des Messmodells der Erfüllung der personellen Anforderungen an die Leistungsfähigkeit wird auf Basis der exploratorischen Faktorenanalyse, wie beabsichtigt, ein Faktor ermittelt, der 83,6 Prozent der Varianz der beiden Indikatoren erklärt. Auch die Konstruktreliabilität, das Cronbach'sche Alpha und die durchschnittlich erfasste Varianz weisen gute Werte auf. Die Faktorladungen liegen mit Werten über 0,9 weit über dem empfohlenen Schwellenwert. Aufgrund der zufrieden stellenden Ergebnisse kann das Messmodell in dieser Form beibehalten werden.

Bei der Überprüfung des Messmodells der Erfüllung der inhaltlichen Anforderungen an die Zusammenarbeit zeichnet sich ein ähnliches Bild ab wie bei der Untersuchung des Messmodells der inhaltlichen Anforderungen an die Leistungsfähigkeit. Durch die Konstruktindikatoren kann kein ausreichend hoher Varianzerklärungsbeitrag erzielt werden. Zudem weist das Cronbach'sche Alpha einen inakzeptablen Wert von 0,230 auf. Die Faktorladung des Items Leistungsnachweis der Agentur hinsichtlich der Umsetzung der Integrierten Kommunikation liegt deutlich unter dem Mindestwert von 0,5. Wird das Item eliminiert, erhöht sich der Varianzerklärungsbeitrag des Konstrukts auf 62,3 Prozent, das Cronbach'sche Alpha steigt ebenfalls deutlich an. Auch im Hinblick auf die weiteren Prüfkriterien werden nun Werte über dem jeweils empfohlenen Schwellenwert erreicht. Entgegen der Erkenntnisse aus den durchgeführten Interviews geht die Erfüllung der inhaltlichen Anforderungen an die Zusammenarbeit aus Sicht von Unternehmen nicht mit dem von der Agentur erbrachten Leistungsnachweis bezüglich der Umsetzung der Integrierten Kommunikation einher.

Die Güteprüfung des Messmodells der organisatorischen Anforderungen an die Zusammenarbeit verzeichnet auch hier eine mangelnde Erfüllung der Prüfkriterien. Die Resultate verweisen auf die Notwendigkeit der Streichung der Indikatoren Vorliegen formalisierter Abstimmungs- und Entscheidungsregeln sowie örtliche Nähe der Agentur zum Unternehmen. Durch die Item-Eliminierung können die Mindestwerte für sämtliche Gütemasse erreicht werden. Die befragten Unternehmen verbinden die Erfüllung der organisatorischen Anforderungen an die Zusammenarbeit offenbar nicht mit diesen Indikatoren. Dies weist darauf hin, dass für Unternehmen eine regelmässige Kooperation/Koordination und ein institutionalisierter Informationsaustausch ausreicht; das Vorliegen formalisierter Abstimmungs- und Entscheidungsregeln ist für eine gelungene Organisation zwischen Unternehmen und Agenturen hingegen nicht von Bedeutung. Im Hinblick auf die Nähe der Agentur zum 
Unternehmen kann dies auf die zahlreichen multimedialen Kommunikationsmöglichkeiten zurückgeführt werden, die ein persönliches Treffen nicht zwingend notwendig machen.

Im Rahmen der Validierung des Messmodells Erfüllung der personellen Anforderungen an die Zusammenarbeit werden die Mindestwerte für die Gütemasse zum Teil ebenfalls nicht erreicht. Die Ergebnisse führen zur Streichung der Items kontinuierlicher Ansprechpartner auf Agenturseite und Flexibilität der Agenturen. Nach Eliminierung der Items werden die empfohlenen Schwellenwerte klar überschritten. Unternehmen, die in personeller Hinsicht eine positive Zusammenarbeit mit Agenturen wahrnehmen, gehen nicht von einem kontinuierlichen Ansprechpartner auf Agenturseite sowie von der Flexibilität der Agenturen, beispielsweise im Hinblick auf Änderungswünsche, aus. Dies lässt sich möglicherweise damit begründen, dass Unternehmen mit Fragestellungen zu personellen Anforderungen an die Zusammenarbeit eher emotionale Aspekte, wie z. B. ein offener, vertrauenswürdiger Umgang oder die Bereitschaft zur Zusammenarbeit mit dem Unternehmen, verbinden, die Items bezüglich des Ansprechpartners bzw. der Flexibilität von Agenturen eher sachlich ausgerichtet sind.

Bei der Untersuchung des Messmodells Umsetzung der Integrierten Kommunikation durch den Einsatz der Agentur ergibt die exploratorische Faktorenanalyse, dass das Konstrukt 59,4 Prozent der Varianz der zugrunde liegenden Indikatoren erklärt. Damit wird die postulierte Eindimensionalität des Konstrukts bestätigt. Zudem fallen die Werte für das Cronbach'sche Alpha, die DEV und die Konstruktreliabilität durchgängig zufrieden stellend aus und verweisen auf hohe interne Konsistenz. Es finden sich durchgängig Faktorladungen von 0,688 und höher, mit T-Werten, die weit über dem geforderten Mindestwert von 1,64 liegen. Die einheitliche Verwendung von Botschaften und Bildelementen in der Zielgruppenansprache auf verschiedenen Marktstufen, die Einhaltung formaler Gestaltungsrichtlinien bei Logos und Slogans, sowie der terminlich abgestimmte Einsatz von Massnahmen und Instrumenten spiegeln demnach die Umsetzung der Integrierten Kommunikation gut wider.

Ferner werden die Determinanten der Umsetzung der Integrierten Kommunikation mithilfe des Fornell-Larcker-Kriteriums auf Diskriminanzvalidität untersucht (vgl. für die Werte Tabelle 5 im Anhang). Es zeigt sich, dass die durchschnittlich erfasste Varianz der meisten Faktoren grösser ist als die quadrierte Korrelation zwischen den Faktoren. Somit liegt Diskriminanzvalidität vor. Eine Ausnahme stellt das Konstrukt inhaltliche Anforderungen an die Leistungsfähigkeit dar, das mit den Konstrukten inhaltliche Anforderungen an die $\mathrm{Zu}$ sammenarbeit und personelle Anforderungen an die Zusammenarbeit nach Fornell-Larcker nicht diskriminiert. Da die Durchführung einer jeweils gemeinsamen exploratorischen Faktorenanalyse jeweils die Zuordnung der Indikatoren zu den ursprünglichen Konstrukten aufzeigt, soll dieses Ergebnis toleriert werden.

Bei der Überprüfung des Strukturmodells offenbart sich folgendes Bild: Von den fünf theoretisch postulierten Pfadkoeffizienten weisen die beiden inhaltlichen Anforderungskonstrukte an Leistungsfähigkeit $(0,225)$ und Zusammenarbeit $(0,333)$ sowie das Konstrukt personelle Anforderungen an die Zusammenarbeit $(0,239)$ einen signifikanten Wert auf. Die Varianzerklärung beträgt 54,3 Prozent, d. h., die Umsetzung der Integrierten Kommunikation durch den Einsatz der Agentur wird zu mehr als der Hälfte von den exogenen Konstrukten erklärt. Die Überprüfung des Stone-Geisser-Kriteriums ergibt einen $\mathrm{Q}^{2}$-Wert grösser Null, die Modellstruktur weist also Prognosegüte auf.

Wie in Abbildung 2 ersichtlich, hat die Erfüllung der inhaltlichen Anforderungen an die Zusammenarbeit den stärksten Einfluss auf die Umsetzung der Integrierten Kommunikation, 
gefolgt von der Erfüllung der personellen Anforderungen an die Zusammenarbeit und der inhaltlichen Anforderungen an die Leistungsfähigkeit. Damit wird die grosse Bedeutung der Erfüllung inhaltlicher Aspekte durch Agenturen deutlich. Die Erfüllung personeller Anforderungen an die Leistungsfähigkeit sowie organisatorischer Anforderungen ist für die Umsetzung der Integrierten Kommunikation offensichtlich nicht von Bedeutung. Tabelle 3 fasst die Ergebnisse die Hypothesenprüfung zusammen.

Hypothesen erfüllt?

Je mehr die Agentur die inhaltichen Anforderungen an die

$H_{1}$ : Leistungsfähigkeit erfüllt, desto stärker erfolgt die Umsetzung der Integrierten Kommunikation durch den Einsatz der AgenJA tur.

Je mehr die Agentur die personellen Anforderungen an die

$\mathrm{H}_{2}$ : Leistungsfähigkeit erfüllt, desto stärker erfolgt die Umsetzung der Integrierten Kommunikation durch den Einsatz der Agen-

NEIN tur.

Je mehr die Agentur die inhaltlichen Anforderungen an die $\mathrm{Zu}$ -

$H_{3}$ : sammenarbeit erfüllt, desto stärker erfolgt die Umsetzung der Integrierten Kommunikation durch den Einsatz der Agentur.

Je mehr die Agentur die organisatorischen Anforderungen an

$\mathrm{H}_{4}$ : die Zusammenarbeit erfüllt, desto stärker erfolgt die Umset-

zung der Integrierten Kommunikation durch den Einsatz der

NEIN

Agentur.

Je mehr die Agentur die personellen Anforderungen an die $\mathrm{Zu}$ -

$H_{5}$ : sammenarbeit erfüllt, desto stärker erfolgt die Umsetzung der

Integrierten Kommunikation durch den Einsatz der Agentur.

Tabelle 3: Ergebnisse der Hypothesenprüfung

\section{Fazit, Implikationen und kritische Würdigung}

Das Ziel des Artikels war es zum einen, diejenigen Anforderungen an Agenturen zu identifizieren, die Unternehmen im Rahmen der Umsetzung einer Integrierten Kommunikation an Agenturen stellen. Zum anderen galt es als weitere Zielsetzung, die Relevanz der Erfüllung dieser Anforderungen für die Umsetzung der Integrierten Kommunikation zu ermitteln.

Für die Identifikation der Anforderungen wurden 20 Interviews mit Unternehmen, die im Rahmen der Integrierten Kommunikation mit Agenturen zusammenarbeiten, befragt. Die Durchführung der Interviews führte zur Identifikation von inhaltlichen und personellen Anforderungen an die Leistungsfähigkeit sowie inhaltlichen, organisatorischen und personellen Anforderungen an die Zusammenarbeit.

Für die Ermittlung der Relevanz der Erfüllung dieser Anforderungen für die Umsetzung der Integrierten Kommunikation wurde eine Strukturgleichungsanalyse durchgeführt. Die Ergebnisse unterstreichen die grosse Bedeutung der Erfüllung der inhaltlichen Anforderungen. So ist insbesondere auf „hard facts“, d. h. auf die Erfüllung inhaltlicher Anforderungen, Wert zu legen. Im Folgenden wird auf Implikationen, die sich aus den Studienergebnissen für Agenturen ableiten lassen, eingegangen. Die Reihenfolge ihrer Darstellung richtet sich nach der ermittelten Stärke der überprüften Wirkungszusammenhänge: 
- Für die Erfüllung der inhaltlichen Anforderungen an die Zusammenarbeit ist es Agenturen anzuraten, sich intensiv mit der Unternehmens- und Kommunikationskultur (Brubn 2009, 346ff.) des betreuenden Unternehmens vertraut zu machen. Für die Agentur gilt es dadurch herauszufinden, welchen Stellenwert die Integrierte Kommunikation im Unternehmen einnimmt und inwieweit diese im Unternehmen bereits besteht. Dies gelingt z. B. durch intensive Gespräche, Unternehmensführungen, Teilnahme an Firmenevents usw. Durch diese Massnahmen erhalten Agenturen die Möglichkeit, einen tieferen Einblick in die Unternehmenstätigkeit, Geschäftsbereiche, Unternehmenswerte, Markenhistorie, der im Unternehmen vorliegenden Definition der Integrierten Kommunikation und v. a. auch in die Ziele des Unternehmens zu erhalten. Zudem ist es empfehlenswert, den Unternehmen stets Zwischenberichte über den Status Quo bei der Integration der Kommunikation abzugeben und das Feedback des Unternehmens entgegenzunehmen, um bei Unzufriedenheit auf Seiten des Kunden Lösungen für Verbesserungen zu finden. Weiterhin ist es von Bedeutung, dass die Agenturen ihren Kunden Vorschläge unterbreiten, wie sie den Stand der Integration im Unternehmen intern kommunizieren, um keine Reaktanzen gegen einen möglichen Mehrbedarf an Ressourcen durch die Integrierte Kommunikation aufkommen zu lassen. Besonders bedeutsam ist es, zu Beginn der Zusammenarbeit ein einheitliches Verständnis des Konzepts der Integrierten Kommunikation festzulegen, um späteren Missverständnissen vorzubeugen.

- Für die Erfüllung der von Unternehmen gestellten personellen Anforderungen an die Zusammenarbeit gilt es für Agenturen, eine klare Rollenverteilung vorzunehmen. Aufgaben, Verantwortlichkeiten und Kompetenzen der beteiligten Personen sowie deren Arbeitsabläufe sind unmissverständlich festzulegen. Ausserdem sind bestimmte Mitarbeitende auf beiden Seiten mit gewissen Entscheidungsbefugnissen auszustatten, um ein flexibles und schnelles Handeln zu ermöglichen.

- Für die Erfüllung der von Unternehmen gestellten inhaltlichen Anforderungen an die Leistungsfähigkeit empfiehlt es sich für Agenturen, sich in ihrer Arbeit an den Merkmalen einer „Integrated Communications Agency“ (ICA) (Duncan/Moriarty 1997) zu orientieren. Die Hauptaufgabe einer ICA liegt in - insbesondere der strategischen - Koordination des gesamten Kommunikationsmix und der Sicherstellung der Konsistenz aller Markenbotschaften, die die Zielgruppen über unterschiedliche Kommunikationskanäle erreichen. Ein wesentliches Merkmal der ICA stellt die Entwicklung medienneutraler Kommunikationsstrategien dar. Dabei wird, neben der Breite an Wissen, auch eine gewisse Tiefe an Spezialwissen vorausgesetzt. Zur Realisierung der Anforderungen bieten sich Teams an, die sich aus Experten verschiedener Kommunikationsfachabteilungen zusammensetzen, sowie regelmässige Trainings, um die Mitarbeitenden mit den Charakteristika der einzelnen Kommunikationsinstrumente vertraut zu machen.

Der vermutete Zusammenhang zwischen der Erfüllung personeller Anforderungen durch die Agenturen und der Umsetzung der Integrierten Kommunikation konnte in Bezug auf die Leistungsfähigkeit nicht nachgewiesen werden. Dies steht im Widerspruch zu den im Vorfeld der Befragung durchgeführten Interviews. Als mögliche Erklärung ist ein sozial erwünschtes Verhalten auf Seiten der Unternehmen anzusehen. Vielfach sind Unternehmen abhängig von den Agenturen, da diese das Copyright für das Design usw. haben. Möglicherweise befürchteten die Unternehmen bei der Befragung, durch ein kritisches Antwortverhalten die Agenturen zu verärgern und dadurch die künftige Zusammenarbeit zu erschweren bzw. zu gefährden. Da aus diesem Grund die Erfüllung der personellen Anforderungen an die Leis- 
tungsfähigkeit dennoch als bedeutsam angesehen werden, sollen hierzu ebenfalls einige Implikationen aufgeführt werden:

- Um die von Unternehmen gestellten personellen Anforderungen an die Leistungsfähigkeit zu erfüllen, gilt es für Agenturen, sich kontinuierlich Wissen zum Konzept der Integrierten Kommunikation anzueignen. Dies kann zum einen durch das Agenturmanagement in Form von Handbüchern, Leitfäden oder Broschüren erfolgen, zum anderen aber auch interaktiv durch Seminare, Workshops usw. Neben der Schaffung von Integrationswissen der Mitarbeitenden ist es des Weiteren erforderlich, eine positive Einstellung zur Integrierten Kommunikation bei den Agenturmitarbeitenden zu generieren. Die Integrationseinstellung betrifft die Frage, ob Mitarbeitende mit dem Begriff der Integrierten Kommunikation positive Assoziationen verbinden oder ablehnende Haltungen aufweisen und nur auf ihre Spezialdisziplin fokussiert sind. Das Agenturmanagement hat z. B. durch Trainings, Schulungen, aber auch durch persönliche Gespräche mit Vorgesetzten dafür zu sorgen, dass die Einsicht, das Verständnis und die positive Einstellung zur Integrierten Kommunikation im Sinne eines Verständnisses für die Gesamtkommunikation gefördert werden.

Der nicht nachgewiesene positive Einfluss der organisatorischen Anforderungen an Agenturen im Hinblick auf die Umsetzung der Integrierten Kommunikation widerspricht den Erkenntnissen aus den qualitativen Interviews. Ein möglicher Grund hierfür ist, dass im Rahmen der Studie Unternehmen in der Schweiz befragt wurden, die oftmals Töchter internationaler Konzerne sind, deren Vorgaben meist auf internationaler Ebene konzipiert werden. Demzufolge sind eine regelmässige Kooperation bzw. Kooperation und ein institutionalisierter Informationsaustausch mit Agenturen von geringerer Bedeutung. Es ist aber anzunehmen, dass die Erfüllung der organisatorischen Anforderungen in kleineren und mittleren nationalen Unternehmen stärker von Relevanz ist. Folgende Implikationen ergeben sich hierbei für die Erfüllung dieser Anforderung:

- Für die Erfüllung der organisatorischen Anforderungen ist es Agenturen anzuraten, einen intensiven und andauernden Informations- und Wissensaustausch mit dem Unternehmen zu pflegen. Besonders empfiehlt sich der Einsatz von Informations- und Kommunikationssystemen im Sinne technischer Hilfsmittel, wie z. B. Email, Audio- und Videokonferenzen, Newsletter oder Workgroup Computing. Zudem bieten sich als persönliche Treffen regelmässig stattfindende Meetings, Workshops oder Events an, oder auch das Einbinden von Agenturmitarbeitenden als Consultants im Unternehmen.

Die vorliegende Studie unterliegt auch einigen Restriktionen. Das Modell zeigt zwar, inwiefern die Erfüllung der Anforderungen an Agenturen zur Umsetzung der Integrierten Kommunikation beitragen, nicht aber, inwieweit sich dies auf vorökonomische (z. B. Kundenverhalten) und ökonomische (Umsatz, Marktanteil) Grössen auswirkt. Eine Modellerweiterung ist künftig um diese Grössen vorzunehmen, so dass für Unternehmen der Agenturbeitrag quantitativ sichtbar gemacht werden kann. Im Rahmen der Studie wurde sich ferner auf Anforderungen von Unternehmen an Agenturen konzentriert. Dies stellt aber lediglich eine Fremdperspektive dar. Von Relevanz ist zudem die Ermittlung einer Eigenperspektive. Hierfür sind die Agenturen nach den Anforderungen, von denen sie vermuten, dass diese von Unternehmen an sie gestellt werden, zu befragen. Zwischen der Eigen- und Fremdperspektive bestehen möglicherweise Abweichungen, die für Missverständnisse zwischen Unternehmen und Agenturen ursächlich sein können. Der vorliegende Datensatz beinhaltet zudem Grossunternehmen aus der Schweiz. Wie bereits aufgeführt, ist anzunehmen, dass sich mit dem 
Einbezug kleinerer und mittlerer Unternehmen weitere ursprünglich vermutete Beziehungszusammenhänge als signifikant erweisen. Dies ist in künftigen Studien zu überprüfen. Zudem beschränkt sich die Untersuchung auf die Schweiz. Insofern ist die Studie auch auf internationaler Ebene durchzuführen, um daraus Vergleiche zu ziehen.

\section{Anhang}

Inhaltliche Anforderungen an die Leistungsfähigkeit

EFA = 42,3\%; Konstruktreliabilität = 0,809; Alpha $=0,714 ; \mathrm{DEV}=0,419$

Items

Faktorladung

T-Wert $(>1,64)$

Angebot von Full-Service-Leistungen/Angebot aller nachgefragten Kommunikationsinstrumente

Vorhandensein von Spezialistenwissen (d. h. tief, weniger breit)

Vorhandensein von Generalistenwissen (d. h. breit, weniger tief)

Schnelligkeit der Agentur in der Umsetzung der Integrierten Kommunikation

Strategische Fähigkeiten hinsichtlich Integrierter Kommunikation

Operative Fähigkeiten hinsichtlich Integrierter Kommunikation (d. h. Kompetenz in Sachen praktischer Vernetzung)

Vollständige Verbindung der eingesetzten Kommunikationsinstrumente

\begin{tabular}{c|c|c|} 
& Faktorladung & T-Wert $(>1,64)$ \\
\hline & 0,518 & 4,795 \\
\hline & 0,588 & 6,523 \\
\hline
\end{tabular}

Wurde auf Basis der Validitätsprüfung eliminiert. Wird hier lediglich der Vollständigkeit halber aufgeführt.

Personelle Anforderungen an die Leistungsfähigkeit EFA $=83,6 \% ;$ Konstruktreliabilität $=0,910 ;$ Alpha $=0,804 ;$ DEV $=0,835$

\begin{tabular}{|c|c|c|}
\hline Items & Faktorladung & T-Wert $(>1,64)$ \\
\hline $\begin{array}{l}\text { Bereitschaft der Mitarbeiter der Agentur, sich im Sinne der } \\
\text { Integrierten Kommunikation für das Unternehmen zu en- } \\
\text { gagieren }\end{array}$ & 0,909 & 35,420 \\
\hline $\begin{array}{l}\text { Bereitschaft der Mitarbeiter der Agentur, sich Wissen über } \\
\text { die Integrierte Kommunikation anzueignen }\end{array}$ & 0,918 & 33,741 \\
\hline \multicolumn{3}{|l|}{ Inhaltliche Anforderungen an die Zusammenarbeit } \\
\hline \multicolumn{3}{|c|}{$\mathrm{EFA}=62,3 \%$; Konstruktreliabilität $=0,867 ;$ Alpha $=0,762 ; \mathrm{DEV}=0,626$} \\
\hline Items & Faktorladung & T-Wert $(>1,64)$ \\
\hline Gleiches Verständnis von Integrierter Kommunikation & 0,856 & 30,027 \\
\hline Kenntnis der Agentur über die Ziele des Unternehmens & 0,831 & 18,669 \\
\hline $\begin{array}{l}\text { Bewusstsein der Agentur über die Wichtigkeit der Inte- } \\
\text { grierten Kommunikation für das Unternehmen }\end{array}$ & 0,871 & 25,852 \\
\hline $\begin{array}{l}\text { Leistungsnachweis der Agentur bezüglich der Umsetzung } \\
\text { der Integrierten Kommunikation (z. B. in Form von Eva- } \\
\text { luierungen, usw.) }\end{array}$ & \multicolumn{2}{|c|}{$\begin{array}{l}\text { Wurde auf Basis der Validitätsprüfung eliminiert. } \\
\text { Wird hier lediglich der Vollständigkeit halber auf- } \\
\text { geführt. }\end{array}$} \\
\hline $\begin{array}{l}\text { Explizite Einbeziehung der internen Kommunikation des } \\
\text { Unternehmens durch die Agentur }\end{array}$ & 0,570 & 5,386 \\
\hline
\end{tabular}




\begin{tabular}{|c|c|c|}
\hline \multicolumn{3}{|l|}{ Organisatorische Anforderungen an die Zusammenarbeit } \\
\hline \multicolumn{3}{|c|}{ EFA $=86,1 \% ;$ Konstruktreliabilität $=0,876 ;$ Alpha $=0,832 ; \mathrm{DEV}=0,780$} \\
\hline Items & Faktorladung & T-Wert $(>1,64)$ \\
\hline $\begin{array}{l}\text { Regelmässige Kooperation/Koordination zwischen Unter- } \\
\text { nehmen und Agentur }\end{array}$ & 0,920 & 9,222 \\
\hline $\begin{array}{l}\text { Regelmässiger, institutionalisierter Informationsaus- } \\
\text { tausch zwischen Unternehmen und Agentur (z. B. Work- } \\
\text { shops, Round-Table-Gesprächen, etc.) }\end{array}$ & 0,844 & 5,743 \\
\hline $\begin{array}{l}\text { Vorliegen von formalisierten Abstimmungs- und Entschei- } \\
\text { dungsregeln zwischen Unternehmen und Agentur (z. B. } \\
\text { Milestones) }\end{array}$ & \multicolumn{2}{|c|}{$\begin{array}{l}\text { Wurde auf Basis der Validitätsprüfung eliminiert. } \\
\text { Wird hier lediglich der Vollständigkeit halber auf- } \\
\text { geführt. }\end{array}$} \\
\hline Örtliche Nähe der Agentur zum Unternehmen & \multicolumn{2}{|c|}{$\begin{array}{l}\text { Wurde auf Basis der Validitätsprüfung eliminiert. } \\
\text { Wird hier lediglich der Vollständigkeit halber auf- } \\
\text { geführt. }\end{array}$} \\
\hline \multicolumn{3}{|l|}{ Personelle Anforderungen an die Zusammenarbeit } \\
\hline \multicolumn{3}{|c|}{$\mathrm{EFA}=58,1 \% ;$ Konstruktreliabilität $=0,757 ;$ Alpha $=0,832 ; \mathrm{DEV}=0,580$} \\
\hline Items & Faktorladung & T-Wert $(>1,64)$ \\
\hline $\begin{array}{l}\text { Gleiche Auffassungen über die Rolle der Agentur im Rah- } \\
\text { men der integrierten Kommunikationsarbeit }\end{array}$ & 0,876 & 24,017 \\
\hline $\begin{array}{l}\text { Bereitschaft zur Abstimmung und Zusammenarbeit mit } \\
\text { dem Unternehmen }\end{array}$ & 0,831 & 13,176 \\
\hline Offener, vertrauenswürdiger Umgang & 0,621 & 5,626 \\
\hline Kontinuierlicher Ansprechpartner auf Seiten der Agentur & \multicolumn{2}{|c|}{$\begin{array}{l}\text { Wurde auf Basis der Validitätsprüfung eliminiert. } \\
\text { Wird hier lediglich der Vollständigkeit halber auf- } \\
\text { geführt. }\end{array}$} \\
\hline $\begin{array}{l}\text { Flexibilität der Agenturen (z. B. Reaktion auf Änderungs- } \\
\text { wünsche) }\end{array}$ & \multicolumn{2}{|c|}{$\begin{array}{l}\text { Wurde auf Basis der Validitätsprüfung eliminiert. } \\
\text { Wird hier lediglich der Vollständigkeit halber auf- } \\
\text { geführt. }\end{array}$} \\
\hline $\begin{array}{l}\text { Zuverlässigkeit der Agentur im Rahmen der integrierten } \\
\text { Kommunikationsarbeit (z. B. das Einhalten von Vorgaben) }\end{array}$ & 0,689 & 6,370 \\
\hline \multicolumn{3}{|c|}{ Umsetzung der Integrierten Kommunikation durch den Einsatz der Agentur } \\
\hline Items & Faktorladung & T-Wert $(>1,64)$ \\
\hline \multicolumn{3}{|c|}{ EFA $=59,4 \% ;$ Konstruktreliabilität $=0,911 ;$ Alpha $=0,885 ; \mathrm{DEV}=0,595$} \\
\hline \multicolumn{3}{|l|}{ Inhaltliche Integration durch den Einsatz der Agentur } \\
\hline $\begin{array}{l}\text { Die Vermittlung von Inhalten erfolgt in einheitlichen Kom- } \\
\text { munikationsbotschaften }\end{array}$ & 0,770 & 15,789 \\
\hline $\begin{array}{l}\text { Die Verwendung einheitlicher Bildelemente wird über ver- } \\
\text { schiedene Kommunikationsinstrumente hinweg eingehal- } \\
\text { ten }\end{array}$ & 0,761 & 12,734 \\
\hline $\begin{array}{l}\text { Die einheitliche kommunikative Ansprache erfolgt für alle } \\
\text { relevanten Zielgruppen (wie z. B. Absatzmittler und End- } \\
\text { kunden) auf verschiedenen Marktstufen }\end{array}$ & 0,777 & 13,434 \\
\hline
\end{tabular}




\begin{tabular}{|l|c|c|}
\hline \multicolumn{2}{|l|}{ Formale Integration durch den Einsatz der Agentur } & \\
\hline $\begin{array}{l}\text { Die Beachtung formaler Gestaltungsrichtlinien erfolgt über } \\
\text { verschiedene Kommunikationsinstrumente hinweg bei } \\
\text { Zeichen und Logos }\end{array}$ & 0,797 & 11,616 \\
\hline $\begin{array}{l}\text { Die Beachtung formaler Gestaltungsrichtlinien erfolgt über } \\
\text { verschiedene Kommunikationsinstrumente hinweg bei Slo- } \\
\text { gans }\end{array}$ & 0,688 & 8,853 \\
\hline Zeitliche Integration durch den Einsatz der Agentur & & \\
\hline $\begin{array}{l}\text { Innerhalb eines Kommunikationsinstrumentes sind die ein- } \\
\text { zelnen Kommunikationsmassnahmen/-mittel zeitlich auf- } \\
\text { einander abgestimmt }\end{array}$ & 0,814 & 18,015 \\
\hline $\begin{array}{l}\text { Der Einsatz verschiedener Kommunikationsinstrumente } \\
\text { wird zeitlich aufeinander abgestimmt }\end{array}$ & 0,784 & 13,466 \\
\hline
\end{tabular}

\section{Tabelle 4: Ergebnisse der Messmodelle}

\begin{tabular}{|c|c|c|c|c|c|}
\hline & $\begin{array}{l}\text { Inhaltliche An- } \\
\text { forderungen an } \\
\text { die Leistungsfä- } \\
\text { higkeit }\end{array}$ & $\begin{array}{c}\text { Personelle Anfor- } \\
\text { derungen an die } \\
\text { Leistungsfähig- } \\
\text { keit }\end{array}$ & $\begin{array}{l}\text { Inhaltliche An- } \\
\text { forderungen an } \\
\text { die Zusammen- } \\
\text { arbeit }\end{array}$ & $\begin{array}{l}\text { Organisatori- } \\
\text { sche Anforde- } \\
\text { rungen an die } \\
\text { Zusammenar- } \\
\text { beit }\end{array}$ & $\begin{array}{c}\text { Personelle An- } \\
\text { forderungen an } \\
\text { die Zusammen- } \\
\text { arbeit }\end{array}$ \\
\hline $\begin{array}{l}\text { Inhaltliche An- } \\
\text { forderungen an } \\
\text { die Leistungsfä- } \\
\text { higkeit }\end{array}$ & 0,419 & & & & \\
\hline $\begin{array}{l}\text { Personelle Anfor- } \\
\text { derungen an die } \\
\text { Leistungsfähig- } \\
\text { keit }\end{array}$ & 0,299 & 0,835 & & & \\
\hline $\begin{array}{l}\text { Inhaltliche An- } \\
\text { forderungen an } \\
\text { die Zusammen- } \\
\text { arbeit }\end{array}$ & 0,516 & 0,423 & 0,626 & & \\
\hline $\begin{array}{l}\text { Organisatorische } \\
\text { Anforderungen } \\
\text { an die Zusam- } \\
\text { menarbeit }\end{array}$ & 0,038 & 0,023 & 0,040 & 0,780 & \\
\hline $\begin{array}{l}\text { Personelle Anfor- } \\
\text { derungen an die } \\
\text { Zusammenarbeit }\end{array}$ & 0,419 & 0,364 & 0,523 & 0,038 & 0,580 \\
\hline
\end{tabular}

Tabelle 5: Überprüfung der Anforderungskonstrukte auf Diskriminanzvalidität 


\section{Literaturhinweise}

Bagozzi, R.P./Fornell, C. (1982): Theoretical Concepts, Measurements, and Meaning, in: Fornell, C. (Hrsg.): A Second Generation of Multivariate Analysis, 2. Aufl., New York, S. 24-38.

Bagozzi, R.P./Phillips, L.W. (1982): Representing and Testing Organizational Theories: A Holistic Construal, in: Administrative Science Quarterly, Vol. 27, No. 3, S. 459-489.

Bagozzi, R.P./Yi, Y. (1988): On the Evaluation of Structural Equation Models, in: Journal of the Academy of Marketing Science, Vol. 16, No. 1, S. 74-94.

Beard, F. (1996): Integrated Marketing Communications: New Role Expectations and Performance Issues in the Client-Ad Agency Relationship?, in: Journal of Business Research, Vol. 37, No. 3, S. 207-215.

Belch, G.E./Belch, M.A. (2009): Advertising and Promotion. An Integrated Marketing Communications Perspective, 8. Aufl., Boston u.a.

Bruhn, M. (2009): Integrierte Unternehmens- und Markenkommunikation. Strategische Planung und operative Umsetzung, 5. Aufl., Stuttgart.

Cathey, A. (1996): Integrated Marketing Communications: A Comparison of Thought and Practice, in: Droge, C./Calantone, R. (Hrsg.): 1996 AMA Educators' Proceedings: Enhancing Knowledge Development in Marketing, Vol. 7, Chicago, S. 113-119.

Caywood, C./Schultz, D./Wang, P. (1991): Integrated Marketing Communications. A Survey of National Consumer Goods Advertisers, unveröffentlichter Forschungsbericht, o.O., S. 1-42.

Chin, W.W. (1998a): Issues and opinion on structural equation modeling, in: MIS Quarterly, Vol. 22, No. 1, S. 7-16.

Chin, W.W. (1998b): The Partial Least Approach to Structural Equation Modeling, in: Marcoulides, G.A. (Hrsg.): Modern Methods for Business Research, Mahwah, S. 295-336.

Chin, W.W./Newsted, P.R. (1999): Structural Equation Modeling Analysis with Small Samples Using Partial Least Squares, in: Hoyle, R.H. (Hrsg.): Strategies for Small Sample Research, Thousand Oaks, S. 307-347.

Churchill Jr., G.A. (1979): A Paradigm for Developing Better Measures of Marketing Constructs, in: Journal of Marketing Research, Vol. 16, No. 1, S. 64-73.

Cornelissen, J.P./Lock, A.R. (2000): Theoretical Concept or Management Fashion? Examining the Significance of IMC, in: Journal of Advertising Research, Vol. 40, No. 5, S. 7-15.

Court, D.C./Gordon, J. W./Perrey, J. (2005): Boosting returns on marketing investment, in: McKinsey Quarterly, Nr. 2, S. 36-47, http://www.mckinseyquarterly.com (Zugriff: 07.07. 2010).

Davidson, S./Ewing, M.T. (1997): Integrated Marketing Communications: An Exploratory Investigation of Industry Practices and Perceptions in Australia, Working Paper Series 9701, Curtin University of Technology, Perth.

Duncan, T.R./Everett, S.E. (1993): Client Perceptions of Integrated Marketing Communications, in: Journal of Advertising Research, Vol. 33, No. 3, S. 30-39.

Duncan, T.R./Moriarty, S.E. (1997): Driving Brand Value. Using Integrated Marketing to Manage Profitable Stakeholder Relationships, New York u.a.

Duncan, T.R./Multhern, F. (2004): A white paper on the status, scope and future of IMC, IMC Symposium co-sponsored by IMC programs at Northwestern University and University of Denver (March). 
Eagle, L./Kitchen, Ph.J. (2000): IMC, Brand Communications, and Corporate Cultures. Client/Advertising Agency Co-ordination and Cohesion, in: European Journal of Marketing, Vol. 34, No. 5/6, S. 667-686.

Elms, S. (2007): The long media tail: Are you wagging it? Or, is it wagging you?, Paper presented at Worldwide Multi-Media Measurement, ESOMAR, June, Dublin.

Fasse, J. (2007): This could be heaven: How to measure media in a fragmented world, Paper presented at Worldwide Multi-Media Measurement, ESOMAR, June, Dublin.

Finne, Å./Grönroos, Ch. (2009): Rethinking marketing communication: From integrated marketing communication to relationship communication, in: Journal of Marketing Communications, Vol. 15, No. 2/3, S. 179-195.

Fornell, C./Larcker, D.A. (1981): Evaluating Structural Equation Models with Unobservable Variables and Measurement Error, in: Journal of Marketing Research, Vol. 18, No. 1, S. 39-50.

Gould, S.J./Grein, A.F./Lerman, D.B. (1999): The Role of Agency-Client Integration in Integrated Marketing Communications: A Complementary Agency Theory-Interorganizational Perspective, in: Journal of Current Issues and Research in Advertising, Vol. 21, No. 1, S. 1-12.

Gronstedt, A. (1996): How Can Agencies Support Integrated Communications, in: Journal of Business Research, Vol. 37, No. 3, S. 201-206.

Herrmann, A./Huber, F./Kressmann, F. (2006): Varianz- und kovarianzbasierte Strukturgleichungsmodelle - Ein Leitfaden zu deren Spezifikation, Schätzung und Beurteilung, in: Zeitschrift für betriebswirtschaftliche Forschung, Jg. 58, Nr. 2, S. 34-66.

Homburg, Ch./Giering, A. (1996): Konzeptualisierung und Operationalisierung komplexer Konstrukte - Ein Leitfaden für die Marketingforschung, in: Marketing ZFP, Jg. 18, Nr. 1, S. 5-24.

Jarvis, C.B./Mackenzie, S.B./Podsakoff, Ph.M./Mick, D.G./Bearden, W.O. (2003): A critical review of construct indicators and measurement model misspecifications in marketing and consumer research, in: Journal of Consumer Research, Vol. 30, No. 2, S. 199-218.

Kerr, G./Drennan, J. (2010): Same But Different - Perceptions of Integrated Marketing Communication Among Marketing Communication Partners in Australia, in: Journal of Promotion Management, Vol. 16, No. 6, S. 6-24.

Kerr, G./Schultz, D./Patti, Ch./Kim, I. (2008): An inside-out approach to integrated marketing communication. An international analysis, in: International Journal of Advertising, Vol. 27, No. 4, S. 511-548.

Kessler, C. (2004): Branding in store: Marketing in the 21st century, in: Journal of Brand Management, Vol. 11, No. 4, S. 261-264.

Kim, I./Han, D./Schultz, D.E. (2004): Understanding the Diffusion of Integrated Marketing Communications, in: Journal of Advertising Research, Vol. 44, No. 1, S. 31-45.

Kitchen, Ph.J./Schultz, D.E. (1999): A Multi-Country Comparison of the Drive for IMC, Journal of Advertising Research, Vol. 39, No. 1, S. 21-38.

Kitchen, Ph.J./Schultz, D.E. (2009): IMC: New horizon/false dawn for a marketplace in turmoil, in: Journal of Marketing Communications, Vol. 15, No. 2/3, S. 197-204.

Kitchen, Ph.J./Kim, I./Schultz, D.E. (2008): Integrated Marketing Communications: Practice Leads Theory, in: Journal of Advertising Research, Vol. 48, No. 4, S. 531-546.

Kitchen, Ph.J./Spickett-Jones, J.G./Grimes, T. (2007): Inhibition of Brand Integration amid Changing Agency Structures, in: Journal of Marketing Communications, Vol. 13, No. 2, S. 149-168.

Kitchen, Ph.J./Brignell, J./Li, T./Jones, G.S. (2004a) The emergence of IMC: A theoretical perspective, in: Journal of Advertising Research, Vol. 44, No. 1, S. 19-30. 
Kitchen, Ph.J./Schultz, D.E./Kim, I./Han, D./Li, T. (2004b): Will agencies ever 'get' (or understand) IMC?, in: European Journal of Marketing, Vol. 38, No. 11/12, S. 1417-1436.

Kliatchko, J. (2005): Towards a new definition of Integrated Marketing Communications, in: International Journal of Advertising, Vol. 24, No. 1, S. 7-34.

Kliatchko, J. (2008): Revisiting the IMC construct. A revised definition and four pillars, in: International Journal of Advertising, Vol. 27, No. 1, S. 133-160.

Low, G.S. (2000): Correlates of Integrated Marketing Communications, in: Journal of Advertising Research, Vol. 40, No. 1, S. 27-39.

Masker, S.J./Somosa, T. (2004): Marketing to teens online, in: McKinsey Quarterly, No. 4, S. 4-9.

Moriarty, S./Mitchell, N.D./Wells, W.D. (2009): Advertising: Principles and Practice, 8. Aufl., London.

Nunnally, J.C. (1978): Psychometric Theory, 2. Aufl., New York.

Schultz, D.E. (2003): Evolving marketing and marketing communication into the twenty-first century, in: Iacobucci, D./Calder, B. (Hrsg.): Kellogg on integrated marketing, Hoboken, NJ, S. VII-XXI (Vorwort).

Schultz, D.E./Barnes, B. (1999): Strategic brand communication campaigns, 5. Aufl., Lincolnwood, IL.

Schultz, D.E./Kitchen, Ph.J. (1997): Integrated Marketing Communications in U.S. Advertising Agencies: An Exploratory Study, in: Journal of Advertising Research, Vol. 37, No. 5, S. 7-18.

Schultz, D.E./Tannenbaum, S.I./Lauterborn, R.F. (1994): Integrated Marketing Communications: Putting It Together and Making It Work, Lincolnwood, IL.

Swain, W.N. (2004): Perceptions of IMC after a Decade of Development: Who's at the Wheel, and How Can We Measure Success, in: Journal of Advertising Research, Vol. 44, No. 1, S. 46-65.

Manfred Bruhn, Prof. Dr., ist Inhaber des Lehrstuhls für Betriebswirtschaftslehre, insbesondere Marketing und Unternehmensführung, an der Wirtschaftswissenschaftlichen Fakultät der Universität Basel, und Honorarprofessor an der Technischen Universität München.

Anschrift: Prof. Dr. Manfred Bruhn, Universität Basel, Wirtschaftswissenschaftliche Fakultät, Lehrstuhl für Marketing und Unternehmensführung, Peter Merian-Weg 6, CH-4002 Basel, Tel.: +41 (0)61/267-32-22, Fax: +41 (0)61/267-28-38, E-Mail: manfred.bruhn@unibas.ch

Verena Batt, Dipl.-Rom., ist wissenschaftliche Mitarbeiterin am Lehrstuhl für Betriebswirtschaftslehre, insbesondere Marketing und Unternehmensführung, an der Wirtschaftswissenschaftlichen Fakultät der Universität Basel.

Anschrift: Dipl.-Rom. Verena Batt, Universität Basel, Wirtschaftswissenschaftliche Fakultät, Lehrstuhl für Marketing und Unternehmensführung, Peter Merian-Weg 6, CH-4002 Basel, Tel.: +41 (0)61/267-28-39, Fax: +41 (0)61/267-28-38, E-Mail: verena.batt@unibas.ch 